\title{
ARTIGOS
}

\section{MONTAIGNE, PHILOSOPHE AU QUOTIDIEN: VIE PRIVÉE ET VIE PUBLIQUE DANS LES ESSAIS}

\author{
Philippe Desan* \\ p-desan@uchicago.edu
}

RÉSUMÉ De concert avec ses fonctions politiques - et cela sur vingt ans -, Montaigne rédigea ses Essais que l'on considère trop souvent comme séparés de ses responsabilités publiques aujourd'hui reléguées à l'arrière plan de sa carrière d'écrivain. Nous voudrions arguer que ses Essais sont indissociables de sa vie publique. Certes, les préoccupations littéraires et politiques de Montaigne changent avec son temps, mais ce qui fonde la forme de l'essai - à savoir un discours profondément inscrit dans le présent - ne permet pas d'établir une différence théorique entre le privé et le public puisque les deux sont pratiquement inséparables quand ils sont réduits à leur unité la plus stricte qui se rapporte essentiellement au quotidien, car dans l'immédiat du vécu, le privé et le public sont pratiquement indissociables. Si une philosophie se dégage indubitablement des Essais, elle a peut-être plus à voir avec la manipulation constante effectuée par Montaigne entre le particulier (vie privée) et l'universel (vie publique) que sur le contenu de tel précepte moral ou dogme des écoles antiques. La temporalité de l'écriture et celle des événements ne sont pas en synchronie dans les Essais et Montaigne joue constamment sur cette image d'un livre privé (hors de toute temporalité historique) alors que les Essais sont conçus comme un objet lui permettant précisément l'accès à la vie publique. Cette philosophie du quotidien et du temps présent qui ressort des Essais ne saurait mettre à l'écart

* University of Chicago. Artigo recebido em 12/11/2011.

KRITERION, Belo Horizonte, no 126, Dez./2012, p. 331-349 
les considérations publiques et historiques qui rythment toujours l'écriture d'une vie bien particulière.

Mots-clés Montaigne, vie privée, vie publique, quotidien.

RESUMO Paralelamente a suas funções politicas - e isso em 20 anos - Montaigne escreveu seus Ensaios frequentemente considerados como separados de suas responsabilidades públicas, atualmente relegadas a um segundo plano em relação à sua carreira de escritor. Gostaríamos de argumentar que seus ensaios são inseparáveis de sua vida pública. Certamente, as preocupações literárias e políticas de Montaigne mudam com o seu tempo, mas o que funda a forma do ensaio - a saber, um discurso profundamente inscrito no presente - não permite estabelecer uma diferença teórica entre público e privado, uma vez que os dois são praticamente inseparáveis quando reduzidos à sua unidade mais estrita, a qual se relaciona principalmente com a vida cotidiana, porque no imediato do vivido, o privado e o público são praticamente indissociáveis. Se uma filosofia emerge, sem dúvida, dos Ensaios, ela talvez tenha mais a ver com a manipulação constante realizada por Montaigne entre o particular (vida privada) e o universal (vida pública) do que com o conteúdo de algum preceito moral ou dogma das escolas antigas. A temporalidade da escrita e a dos eventos não estão em sincronia nos Ensaios e Montaigne opera constantemente sobre a imagem de um livro privado (fora de toda temporalidade histórica), enquanto os Ensaios são concebidos como um objeto, permitindo-lhe precisamente o acesso à vida pública. Essa filosofia do cotidiano e do tempo presente que promana dos Ensaios não afasta as considerações públicas e históricas que sempre dão ritmo à escrita de uma vida muito particular.

Palavras-chave Montaigne, vida privada, vida pública, cotidiano.

La critique des $\mathrm{XX}^{\mathrm{e}}$ et $\mathrm{XXI}^{\mathrm{e}}$ siècles s'est presque toujours efforcée d'établir une séparation franche entre la vie privée et la vie publique de l'auteur des Essais. Certes, cette distinction est fondée sur une déclaration de Montaigne lui-même : " Le Maire et Montaigne ont tousjours esté deux, d'une separation bien claire » (III, 10, 1012)1. Il faut pourtant se méfier 
d'une telle assertion et éviter de prendre l'auteur des Essais trop à la lettre. Remarquons d'abord que cet aveu est assez tardif dans la carrière publique de Montaigne puisqu'il fut très certainement rédigé après 1585, c'est-à-dire après ses deux mandats à la mairie de Bordeaux. Rappelons ensuite que, entre 1572 et 1588, Montaigne possédait des ambitions politiques - c'est-àdire publiques - qu'il tenta de satisfaire à plusieurs reprises. Après avoir cédé sa charge de parlementaire à Florimond de Raemond en 1570, et suite à une courte retraite sur ses terres, il envisagea dans un premier temps une carrière dans la diplomatie avant de se retrouver à la tête de la quatrième ville de France - après Paris, Lyon et Rouen - en 1581. On sait enfin qu'il fut chargé de plusieurs missions de rapprochement entre Henri III et le roi de Navarre dans un climat politique et religieux des plus tendus ${ }^{2}$.

De concert avec ces fonctions politiques - et cela sur vingt ans -, Montaigne rédigea ses Essais que l'on considère trop souvent comme séparés de ses responsabilités publiques aujourd'hui reléguées à l'arrière plan de sa carrière d'écrivain. En fait Montaigne ne fit jamais carrière en tant qu'auteur et nous voudrions ici arguer que ses Essais sont indissociables de sa vie publique. D'un point de vue de la temporalité, comme nous allons le voir, ces distinctions sont d'ailleurs purement formelles et ne résistent pas aux exigences sociales et politiques du présent qui fait agir ou écrire l'essayiste. Certes, les préoccupations littéraires et politiques de Montaigne changent avec son temps, mais ce qui fonde la forme de l'essai - à savoir un discours profondément inscrit dans le présent - ne permet pas d'établir une différence théorique entre le privé et le public puisque les deux sont pratiquement inséparables quand ils sont réduits à leur unité la plus stricte qui se rapporte essentiellement au quotidien. Car dans l'immédiat du vécu, le privé et le public sont pratiquement indissociables. Ces termes n'acquièrent un sens qu'avec le recul du temps et à partir d'un effet plus théorique que pratique.

Il a fallu attendre les travaux de Alphonse Grün (1855) pour redécouvrir tout un pan caché de la vie publique de Montaigne ${ }^{3}$. Depuis cette étude d'ailleurs assez mal reçue à son époque, il faut reconnaître que l'aspect public de la pensée de Montaigne n'a guère fait fortune. On préfère aujourd'hui l'image du philosophe retiré du monde, détaché, voire impassible devant l'actualité de son temps et les affres de la vie en société. Cette approche qui met en avant l'hyper subjectivité de l'auteur au détriment de sa sociabilité s'intéresse notamment à la quête de la sagesse, une introspection perpétuelle

2 Voir notamment Donald Frame, Montaigne. Une vie, une œuvre, Paris, H. Champion, 1994.

3 Alphonse Grün, La Vie publique de Michel Montaigne. Etude biographique, Genève, Slatkine, 1970. 
où le sujet, en autarcie parfaite, se suffit à lui-même pour interpréter l'homme particulier et privé dans sa dimension universelle. Au dire des partisans de ce clivage entre le privé et le public chez Montaigne, les guerres de religion et le massacre de la Saint-Barthélemy auraient même créé les impulsions nécessaires à ce détachement avoué vis-à-vis des événements du dernier quart $\mathrm{du} \mathrm{XVI}^{\mathrm{e}}$ siècle. Ne pas commenter son époque - ou du moins passer sous silence une partie des grands événements historiques et politiques de son temps - serait en quelque sorte la preuve irréfutable de cette " separation » nécessaire entre le privé et le public alléguée par l'auteur des Essais. Parler de soi reviendrait alors à présenter la forme universelle de l'homme en général, un homme coupé de sa propre historicité.

Naquit alors le mythe de l'ontologie montaignienne et de sa récupération dans la philosophie moderne. Pour un livre qui réclame sans cesse sa consubstantialité avec un corps de chair et de sang, il est d'ailleurs bien malaisé de faire la part des choses entre l'homme public et l'homme privé qui se confondent fréquemment, quoi qu'en dise Montaigne ${ }^{4}$. C'est peut-être pourquoi cette distinction si à la mode de nos jours entre vie publique et vie privée est totalement absente dans les premières biographies de Montaigne qui considéraient vie privée et vie publique comme un tout indissociable ${ }^{5}$. Le maire et Michel ne pouvaient être qu'un, car, aux XVII ${ }^{e}$ et XVIII ${ }^{e}$ siècles, il aurait été étrange de voir en Montaigne autre chose qu'un homme tout court, un homme avec une dimension sociale et politique inséparable de ses réflexions plus personnelles sur ses habitudes de table par exemple. Certes, même si Montaigne parle trop souvent de lui, Pascal et consorts comprirent rapidement que le moi montaignien pouvait s'imposer comme modèle pour d'autres. C'est ainsi que Montaigne fut rapidement considéré comme un moraliste, dans le sens prescriptif du terme, c'est-à-dire un philosophe du social et du public; les comportements idiosyncrasiques et particuliers ne se concevant que dans une logique de l'exemplarité pour les autres. On pense plus particulièrement aux premiers lecteurs qui virent en Montaigne un pédagogue.

Depuis le début du $\mathrm{XX}^{\mathrm{e}}$ siècle, on a lentement transformé Montaigne en philosophe de la subjectivité et de la vie privée. Considéré comme le

4 Jean Balsamo a étudié ce rapport problématique, voir " 'Le plus grand bien que j'atande de cete miene charge publique' : Montaigne entre vie publique et vie privée ", Nouveau Bulletin de la Société des Amis de Montaigne, $\mathrm{n}^{\circ}$ IV, 2008, p. 359-375.

5 Voir nos études, "Les protobiographies de Montaigne aux XVIle et XVIIle siècles: Scévole de SainteMarthe, Bouhier, Dom Devienne, Talbert, La Dixmerie, Lamontagne, Bourdic-Viot », Montaigne Studies, vol. XX, 2008, p. 11-19; et "Les limites d'une biographie: le cas de Montaigne ", Cahiers parisiens / Parisian Notebooks, $n^{\circ} 4,2008$, p. 575-587. 
précurseur de Descartes et donc de la philosophie moderne, Montaigne se serait sciemment coupé des événements de son temps pour mieux durer à travers les siècles. Tout comme si Montaigne n'avait jamais existé au quotidien mais avait ressenti le besoin d'appartenir à une histoire de la philosophie moderne dont il se serait voulu le précurseur. Cette projection dans le devenir philosophique fait défaut dans les Essais, car Montaigne se situe pleinement dans son époque. Le célèbre retrait dans son château n'a rien à voir avec une possible abdication de la vie sociale. Enfermé dans sa tour, on avance souvent que Montaigne aurait ainsi créé une distance critique nécessaire pour mieux juger la société et ses contemporains, établissant ainsi une distance temporelle. Tout comme si le temps s'arrêtait pour lui, comme par magie, dès qu'il passait à la rédaction de ses Essais. Bref, le privé (universel, atemporel) l'aurait finalement emporté sur le public (ponctuel, quotidien) et c'est pour cette raison que Montaigne serait devenu un grand écrivain.

Selon cette même logique, on a également vu en Montaigne le premier intellectuel de la modernité à partir de la définition proposée par Jean-Paul Sartre : un savant est celui qui parle de ce qu'il connaît, un intellectuel est celui qui " se mêle de ce qui ne le regarde pas " ${ }^{6}$. Les Essais seraient donc le reflet d'une attitude plutôt que d'une expertise. Montaigne rejette en effet l'expertise au profit de l'expérience subjective. Si l'homme public acquiert une fonction ou une charge grâce à des connaissances approfondies sur un sujet précis et au moyen de compétences réelles ou imaginées lui conférant une autorité, l'homme privé conserve quant à lui le droit de donner son opinion sur tous les sujets sans exception. Cette image d'un auteur qui se mêle de tout à partir de sa propre expérience colle à la peau de Montaigne depuis le $\mathrm{XVII}^{\mathrm{e}}$ siècle. La vie privée (à partir d'expériences singulières) aurait remplacé la vie publique comme fondement de la morale. Il nous semble cependant important de remettre en question cette soi-disant victoire de la vie privée chez Montaigne et, presque un siècle et demi après Grün, de s'interroger de nouveau sur le rapport entre le privé et le public non seulement dans les Essais mais aussi dans la vie de Montaigne.

Un peu comme pour l'abbaye de Thélème chez Rabelais, la porte d'entrée des Essais affiche une inscription qui vise à justifier et orienter la lecture de 
l'ouvrage : «C'est icy un livre de bonne foy, lecteur. Il t'avertit dès l'entrée, que je ne m'y suis proposé aucune fin, que domestique et privée. Je n'y ai eu nulle consideration de ton service, ny de ma gloire » (Au lecteur). Voilà qui est clair : l'auteur tient résolument les considérations civiles et publiques à l'écart de son livre. Ni serviteur ni maître, sans préoccupation aucune pour sa renommée ou sa gloire - c'est du moins ce que Montaigne soutient -, les Essais n'auraient pour but que de laisser un portrait privé pour ses proches. Tout comme si sa présence et l'expérience que les autres peuvent avoir de lui dans le quotidien ne suffisaient pas ou ne correspondaient pas à la réalité. Montaigne croit-il vraiment que sa famille et ses amis le connaissent si mal pour découvrir dans son texte un autre homme, différent de celui qu'ils côtoient ? Et si ce n'est pas le cas, à quoi sert alors cet ouvrage ? On pourrait bien sûr arguer qu'un portrait est nécessaire au souvenir des hommes illustres et des grands écrivains. Mais il faut ici se situer au moment de la première édition des Essais en 1580. L'idée de postérité est à l'époque une absurdité puisque Montaigne n'a absolument rien accompli : il a récemment abandonné sa carrière de parlementaire et n'a pas encore acquis une dimension publique et politique qui lui permettrait de rédiger des mémoires retraçant le parcours d'une carrière riche et accomplie ${ }^{7}$. Le genre des Essais tel qu'il est conçu en 1580 offre en fait peu d'éléments pour comprendre la postérité de Montaigne, du moins à partir de critères littéraires ou philosophiques. A moins bien entendu que ce livre ait été conçu à d'autres fins, non pas tant personnelles mais plutôt politiques.

On sait que Montaigne commença à rédiger ses Essais en 1571-1572, lors d'un premier « retrait du monde » de courte durée. Durant l'hiver 1571, il fit aménager sa tour à Montaigne et se donna tous les moyens (sentences sur les poutres de sa « librairie », rayonnages avec les livres hérités de La Boétie, devises peintes sur divers murs de sa tour, etc.) pour devenir auteur. Ce moment qui déclencha très certainement l'écriture chez Montaigne est littéralement inscrit sur une paroi intérieure de sa tour. Cette période de transition entre le monde du Palais (où Montaigne fréquentait les robins du Parlement de Bordeaux) et le monde des palais (où Montaigne jouera les courtisans parmi les princes de la haute noblesse) est censée marquer sa « retraite » de la vie publique et exprimer sa lassitude (plus certainement sa déception vis-à-vis de

7 Sur le genre des mémoires dans son rapport à l'essai, nous renvoyons à notre étude, "Des Mémoires de Commynes aux Essais de Montaigne: réflexion sur des genres," in Philippe de Commynes (1511-2011): droit, écriture, deux bâtisseurs de la souveraineté, dir. Joël Blanchard, Genève, Droz, 2011, p. 285-300. 
sa carrière de magistrat) et son désir de passer le reste de sa vie « dans le sein des doctes Vierges ».

Mais ne nous y trompons pas, cette transition fut relativement brève et les sirènes politiques succédèrent une fois de plus au repli de la vie privée. Montaigne n'avait pas encore quarante ans. En octobre 1571, un peu plus d'un an après avoir mis fin à sa carrière de robin, et à la même époque où il concevait le projet d'écrire quelques essais pour encadrer un texte politique de La Boétie, il reçut une lettre de Charles IX l'informant qu'il venait d'être fait chevalier dans l'ordre de Saint-Michel. Récompense assez extraordinaire si l'on considère que Montaigne n'avait encore rien accompli à cette date ; il n'appartenait pas non plus à la haute ou à la moyenne noblesse et était bien loin d'être un personnage connu sur le plan national, voire même régional. Son passage au Parlement de Bordeaux pourrait même être considéré comme un échec dans la mesure où il ne fut jamais en passe d'accéder à la Grand' Chambre $^{8}$. Il faut donc interpréter ce titre exceptionnel qui venait de lui être conféré comme le signe d'une volonté politique de ses patrons (le marquis de Trans et plus généralement la famille de Foix) qui comptaient bien sur ce gentilhomme gascon pour occuper un espace politique redéfini dans une région qui était considérée comme stratégique aussi bien pour le parti royal que pour les réformés. Les Foix se voulaient plus conciliateurs qu'ils ne l'avaient été sous Charles IX et Montaigne jouera le rôle que ses patrons attendaient de lui. Ses Essais occuperont une place essentielle dans son cheminement vers les sphères du pouvoir royal.

Peu de temps après cette « entrée en noblesse », Montaigne fut également nommé gentilhomme ordinaire de la chambre du roi Charles IX en 1573. Ce titre est plus une récompense d'honneur qu'une charge réelle. C'est en effet un office inférieur qui ne demande aucune résidence ou obligation particulière. Les grands officiers de la maison du roi représentaient les vrais officiers. Simplement nommés et non pourvus par lettres patentes, ces petits officiers remplaçaient en fait les valets de chambre, titre désuet et considéré « fort vil » comme le remarque Charles Loyseau dans son traité des Offices. Loyseau décrit le titre et la fonction de ces petits officiers royaux : « La prolifération des aumôniers sous Henri II et des gentilshommes ordinaires de la chambre du roi sous Charles IX ont pour but de s'attacher le service

8 Sur ce point, voir Nussy Saint-Saens, "Montaigne au Parlement de Bordeaux ", Revue historique de Bordeaux et du département de la Gironde, nouvelle série II, 1953, p. 119-135 ; Georges Hubrecht, « Montaigne juriste », in IV Centenaire de la naissance de Montaigne, Bordeaux, Delmas, 1933, p. 239-298. 
de nobles $»^{9}$. Loyseau rend compte du nombre croissant de ces officiers qui « se trouvèrent incontinent en nombre effréné, notamment sous le feu Roy Henry III $»^{10}$. Leur nombre était si élevé (plus de cent cinquante) qu'ils ne recevaient plus aucun gage et n'étaient plus que des officiers honoraires. $\mathrm{Ce}$ fut le cas de Montaigne qui se vante de ne pas être rétribué pour sa charge. Plusieurs cas de « gentilshommes » non issus de la noblesse sont rapportés à cette époque. Ces litiges sur l'origine nobiliaire des gentilshommes de la chambre du roi entraînèrent des réclamations durant les années 1570. L'article 259 de l'ordonnance de Blois de 1579 stipulait par exemple qu' « aucun ne sera receu aux estats de gentilshommes de nostre chambre qu'il ne soye noble de race ; et si aucuns s'en trouvent qui soient de ladite qualité, y sera par sa Majesté pourvu d'autres en leur place ». Aux États Généraux de 1588, la noblesse se plaignit de la non application de cette ordonnance. Peut-être pour prendre les devants sur de telles accusations, Montaigne ne manquera pas d'indiquer sur les pages de titre des deux premières éditions des Essais (1580, 1582) qu'il appartient bien à la noblesse de race ${ }^{11}$. Il faut sur ce sujet rappeler que, lors de sa réélection à la mairie de Bordeaux en 1583, l'appartenance de Montaigne à la noblesse fit l'objet d'une réclamation par les représentants de la noblesse auprès de la jurade de Bordeaux. Le roi rejettera cette plainte et Montaigne fut donc renouvelé pour un second mandat. Comme on le voit, la rédaction et la première publication des Essais doivent être replacées dans leur contexte social et politique immédiat. La vie privée de Montaigne se confond immanquablement avec la vie publique de ceux qui l'entourent et lui permettent de se lancer à son tour en politique. Intrinsèquement liée aux Essais - mais aussi à la publication des œuvres de La Boétie en 1571 - la vie publique de Montaigne émerge en filigrane dans un projet d'écriture qui varie dans le temps en fonction d'espoirs, d'attentes, de déceptions et de rebondissements politiques.

Le portrait d'un gentilhomme, fervent lecteur des Anciens et de l'Antiquité païenne, doit aussi accorder une place de choix aux valeurs nobiliaires de son temps. Par exemple, un seigneur a l'obligation de guerroyer au service de son souverain. Ainsi, le 11 mai 1574, Montaigne, en qualité de gentilhomme du roi, rejoint l'armée royale en Poitou. Les années qui s'étendent de 1570

9 Charles Loyseau, Du Droict des offices, in CEuvres de Charles Loyseau, Genève, Estienne Gamonet, 1636, Livre IV, chapitre III, p. 579.

10 Ibid.

11 Ainsi, dans les éditions de 1580 et 1582 l'accent est mis sur le mot « MESSIRE », en lettres capitales de taille démesurée par rapport au reste du titre. Notons pourtant que ces titres disparaissent lors de l'édition parisienne de 1588 qui indique uniquement « Essais de Michel seigneur de Montaigne ». 
à 1574 sont marquées par ce que l'on pourrait définir comme la fabrication d'un curriculum vitae de bonne noblesse. Les fréquentations directes et épistolaires des grands seigneurs et autres hommes de pouvoir se multiplient de façon accélérée et, en moins de quatre ans, Montaigne effectue un parcours des plus rapides dans le milieu politique de sa région. Suivant l'expression anglaise, il était devenu un made man et, propulsé à l'avant-scène politique de la Guyenne, sa visibilité publique se trouva très largement accrue. Peu de temps après, le duc de Montpensier le chargea de négocier avec le Parlement de Bordeaux, un milieu qu'il connaissait bien de par sa carrière antérieure. On a démontré de quelle façon Montaigne n'aurait pu acquérir une telle visibilité politique sans de puissants parrains, les marquis de Trans et la famille de Foix ${ }^{12}$. Pourtant, le décès de Charles IX, le 30 mai 1574, posa un véritable problème pour Montaigne et ses appuis politiques. Même si Henri III avait très certainement rencontré Montaigne lors de son séjour de la cour à Bordeaux en 1565, tout restait néanmoins à refaire. Montaigne développa alors une stratégie d'approche de la cour qui n'est pas inhabituelle pour son temps. La publication d'un ouvrage privé lui donnerait l'occasion d'obtenir une entrevue royale. C'est dans ce contexte qu'il faut à notre avis concevoir la première publication des Essais et la remise d'un exemplaire en mains propres au roi à Saint-Maur-des-Fossés en juillet 1580.

En 1580, le livre de Montaigne sert de recommandation et facilite son entrée à la cour. La nouveauté des Essais est précisément d'établir une homologie parfaite entre l'homme et l'œuvre, la vie privée et les compétences publiques : une comparaison que Montaigne ne manquera pas d'évoquer devant le roi. Le livre de Montaigne constitue bien une offre de service, au niveau de la fidélité et de l'amitié bien particulière à cette époque associée à la fonction de conseiller ${ }^{13}$. À en croire La Croix du Maine, le roi aurait même complimenté Montaigne sur son ouvrage. Ce dernier aurait alors répondu : « Sire $[\ldots]$ il faut donc nécessairement que je plaise à votre Majesté, puisque mon Livre lui est agréable, car il ne contient autre chose qu'un discours de ma vie et de mes actions $\gg{ }^{14}$. On comprend dès lors mieux cette stratégie littéraire qui vise à établir une homologie parfaite entre le livre et l'auteur. Si le livre a séduit le roi, c'est que Montaigne plaît au roi. Il lui a plu en tant qu'homme et il est désormais possible d'être considéré comme serviteur politique ou

12 Voir Jean Balsamo, «Un gentilhomme et ses patrons : remarques sur la biographie politique de Montaigne », in Montaigne politique, éd. P. Desan, Paris, H. Champion, 2006, p. 223-242.

13 Alain Legros, « Montaigne, son livre et son roi », Studi Francesi, vol. XLI, fasc. II, 1997, p. 259-274.

14 François de La Croix du Maine, Bibliotheque françoise, Paris, Abel L’Angelier, 1584, p. 328. 
diplomatique ${ }^{15}$. En 1580, le livre sert d'introduction pour une carrière qui se situe non pas dans l'écriture mais bien dans le domaine du politique et donc de la vie publique.

Entre temps, en janvier ou février 1576, et comme pour confirmer sa noblesse, Montaigne avait fait frapper un jeton où se lisaient sur une face ses armes entourées du collier de l'ordre de Saint-Michel et la légende " Michel Seigneur de Montaigne » et, au revers, une balance aux plateaux horizontaux avec la légende « je m'abstiens ». C'est en fait là une devise des plus appropriées pour un négociateur entre deux partis qui s'affrontent ou encore pour un ambassadeur qui rend compte d'une situation et applique les ordres qui lui sont donnés par le souverain à qui il est censé rapporter sans pour autant émettre de jugement personnel ${ }^{16}$.

Le 29 novembre 1577, par lettres patentes, Montaigne est fait gentilhomme de la chambre de Henri de Navarre. Montaigne nous dit ne pas avoir été informé de cette nomination. Apparemment ses protecteurs faisaient sa carrière sans peut-être le consulter, cela des deux côtés du spectre politique de l'époque : aussi bien dans le milieu catholique que dans le parti protestant. D'un point de vue politique, cette décision qui fait de Montaigne un gentilhomme de la cour de Navarre se comprend car le Béarnais essayait à cette époque d'élargir sa clientèle parmi les gentilshommes catholiques. Il recherchait une légitimité qui ne dépendait pas uniquement des clivages confessionnels habituels. C'est à partir de ce moment que la carrière publique de Montaigne offre un certain intérêt pour Henri III, car Montaigne tient désormais le rôle d'intermédiaire politique idéal tout en présentant des garanties de «bon catholique ». Sa foi ne fait aucun doute et l'on ne peut pas non plus lui reprocher d'être proche du parti des Politiques considérés comme dangereux puisqu'ils prônaient trop ouvertement la négociation et le compromis avec les réformés.

15 Nous avons argué ailleurs qu'au moment de la publication de ses Essais en 1580 Montaigne envisageait de faire carrière dans la diplomatie. II comptait en fait remplacer Louis Chasteigner, sieur d'Abain et de La Rochepozay, comme ambassadeur de France à Rome. Chasteigner avait réaffirmé à plusieurs reprises - dès 1579 - son désir de retrouver son pays et les siens et Montaigne conçut alors son voyage en Italie (en fait son séjour à Rome) pour attendre sur place une nomination qui ne viendra jamais. Sur les circonstances de cette carrière diplomatique ratée, voir notre étude, " L'appel de Rome ou comment Montaigne ne devint jamais ambassadeur ", in Chemins de l'exil, havres de paix. Migrations d'hommes et d'idées au XVle siècle, dir. Jean Balsamo et Chiara Lastraioli, Paris, H. Champion, 2009, p. 229-259.

16 Cette notion est par exemple défendue dans les premiers Essais de 1580, notamment dans le chapitre « Un traict de quelques ambassadeurs » où Montaigne précise que « l'office du serviteur [est] de fidelement representer les choses en leur entier, comme elles sont advenües, affin que la liberté d'ordonner, juger et choisir demeurast au maistre » $(I, 17,73)$. L'ambassadeur quant à lui s'abstient de tout jugement ! Cette position peu « montaignienne » sera bien entendu contredite après 1588, époque à partir de laquelle l'auteur des Essais se fait une toute autre idée des diplomates et des conseillers politiques en général. 
La résidence géographique de Montaigne durant ces années lui donne un avantage certain. Les circonstances particulières d'une vie privée se transforment alors en atouts dans l'échiquier public non seulement de la Guyenne mais aussi, de façon plus générale, au niveau de l'Aquitaine. Montaigne fait figure d'intermédiaire idéal entre Henri III et Henri de Navarre de par sa proximité - non pas tant géographique mais aussi intellectuelle - de la cour de Navarre. Mais Montaigne se conçoit avant tout comme un fidèle serviteur du roi. S'il existe politiquement à l'échelon national, ce ne peutêtre qu'à la faveur de Henri III et de Catherine de Médicis. Ses fréquents déplacements à Paris lui permettent de rester proche de la cour et du centre politique autour duquel il gravitera jusqu'en 1588. Nouvellement « anobli », sans pour autant être trop fortement associé aux courtisans, et sans afficher une ardeur religieuse qui caractérise la plupart de ses contemporains, Montaigne va se faire une place de "centriste ». Il écoute, rapporte et ne donne jamais l'impression de prendre ouvertement parti. Conscient de cette qualité recherchée pour les ambassadeurs, il prend son rôle au sérieux et envisage de faire une carrière où son flair politique pourrait servir la cause royale. On retrouve pour cette raison de nombreux échos de cette image de « tendre negotiateur » dans les premiers Essais. C'est durant cette période charnière qui s'étend de 1574 à 1580 que Montaigne tira son épingle du jeu et se fit une place dans l'échiquier politique régional.

Quand on considère le contexte social et politique de la rédaction des Essais, on comprend alors assez mal pourquoi les Essais devraient être considérés comme un ouvrage qui met en scène la vie privée et gomme la vie publique. Ce n'est pas du tout l'impression qui se dégage de la première édition des Essais publiée chez Simon Millanges en 1580. Nous avons vu comment le contexte politique de la rédaction des premiers essais entre 1572 et 1580 laissa une marque profonde sur la conception, la rédaction et la publication du livre de Montaigne. Vie privée et vie publique ne pouvaient être séparées que de façon artificielle par Montaigne lui-même, selon une logique qui, à notre avis, reflète une stratégie nécessitant un commentaire.

L'avis qui ouvre le livre des Essais s'adresse à un lecteur générique, sans nom, un lecteur qui n'est pas nommément un ami ou un membre de sa famille. Il existe donc une contradiction entre le lecteur idéal et avoué (les parents et amis) et le lecteur réel non encore connu, mais que Montaigne peut néanmoins imaginer par le contenu même de ses premiers essais. Mentionnons 
également que la mise en scène de la vie privée apparaît tout juste après une page de titre qui, elle, met en avant les titres publics de l'auteur (« Messire », « chevalier », " gentilhomme », ou encore " maire » et « gouverneur » en 1582). Cette opposition apparente présentée dans l'avis au lecteur disparaît pourtant quand on considère les chapitres qui suivent. Passons donc le seuil du livre... Que découvre-t-on ensuite ? Cinquante-sept chapitres qui relèvent pour la plupart de lieux communs et de sujets qui expriment en majorité une dimension politique et publique d'actualité en cette fin du XVI ${ }^{\mathrm{e}}$ siècle. Ces topoi politiques développés dans la première édition des Essais sont aux antipodes des fins personnelles invoquées par l'auteur dans son avis au lecteur. Donnons quelques exemples : « Si le chef d'une place assiégée doit sortir pour parlementer » (I, 5), « L'heure des parlemens dangereuse » $(\mathrm{I}, 6)$, « Ceremonie de l'entrevue des roys » (I, 13), « Un traict de quelques ambassadeurs » (I, 17), « De la coutume et de ne changer aisément une loi receüe » (I, 23), « D’un defaut de nos polices » $(\mathrm{I}, 35)$, « Des loix somptuaires » $(\mathrm{I}, 43)$, « De la bataille de Dreux » (I, 45), et encore bien d'autres chapitres qui sont loin de la peinture du moi annoncée par Montaigne.

Comme nous l'avons suggéré, dans son avis au lecteur, Montaigne crée l'illusion d'un désengagement et d'une séparation marquée entre la vie publique et la vie privée alors qu'un nombre important des chapitres des premiers Essais mettent au contraire l'accent sur la gouvernance des états, les stratégies militaires, les entretiens diplomatiques ou encore les valeurs morales de la noblesse d'épée. En 1580 la stratégie de Montaigne est de plaire au roi en mettant l'accent sur ses qualités personnelles (modération, candeur, affabilité, honnêteté) qui sont applicables dans la vie publique. Montaigne met aussi en avant ses défauts, comme il le souligne dans l'avis au lecteur : « Mes defauts s'y liront au vif, et ma forme naïfve ». Cette transparence de caractère connaît pourtant les limites du décorum et de la bienséance protocolaire : « autant que la reverence publique me l'a permis » comme le précise Montaigne. Un tel jugement des limites sociales représente une qualité désirable pour les charges publiques. Le livre de Montaigne est donc bien un portrait privé, non pas tant pour ses amis ou ses parents, mais plutôt pour le roi, celui même pour qui une telle distinction entre privé et public n'existe pas. L'auteur des Essais peint l'image d'un homme dévoué et fidèle faisant à la fois preuve de discernement (en public) et de naïveté (pour son lecteur, le roi) dans les affaires militaires et diplomatiques. Au XVI siècle les ambassadeurs sont recrutés à partir des critères de loyauté et d'allégeance, dans un rapport d'homme à homme et de confiance absolue. Il s'agissait pour Montaigne d'exhiber quelques particularités de sa vie privée afin que l'on puisse associer 
l'homme et le livre. Une entrevue royale ne pouvait suffire et le livre possède de surcroît l'avantage de révéler l'homme plus en profondeur.

Se présenter à Henri III comme être privé en qui il peut avoir confiance pour défendre les intérêts du royaume et de la couronne explique la présence de ces deux niveaux (privé et public) en apparence contradictoires mais en vérité complémentaires dans les Essais de 1580 : la peinture de la vie privée permettant de décrocher une charge publique. Dans le cas de Montaigne c'est-à-dire dans l'optique d'une nouvelle carrière convoitée -, la connaissance de la vie privée des futurs ambassadeurs est une condition essentielle à leur nomination par le pouvoir royal. Le premier avis au lecteur de l'édition de 1580 ne pouvait cependant pas identifier directement son public (royal) et Montaigne préféra se retrancher derrière la présentation d' « un livre de bonne foy » et consubstantiel à son auteur. Son insistance sur le fait que ce livre n'a aucunement pour but de « rechercher la faveur du monde » est assez révélatrice d'une préoccupation constante qui met en avant ses intérêts tout en les niant.

Loin d'exprimer le fondement des Essais, la séparation effectuée par Montaigne après 1588 - dans le troisième livre des Essais et surtout dans les allongeails de l'Exemplaire de Bordeaux - entre vie publique et vie privée arrive tard dans sa réflexion et fait suite à ce que nous pourrions appeler une désillusion de la sphère publique. Après 1588, Montaigne n'aura plus d'autre choix que de théoriser ce qu'il faut bien considérer comme un échec politique. Son avis au lecteur n'a pas besoin d'être modifié dans la mesure où il reflète désormais un constat. C'est là toute l'ironie de ce texte que l'auteur des Essais ne modifiera pratiquement pas d'une édition à l'autre. Certes Montaigne pèse toujours ses mots et les Essais sont l'antithèse du pamphlet. Son livre n'a jamais eu pour but d'être proche des disputes et conflits de son temps. La proximité des événements n'occupe jamais une place de choix dans ses réflexions et ses jugements. Il préfère tirer la plupart de ses exemples de l'Antiquité, mais, comme Machiavel, il trouve fréquemment des similitudes historiques ou politiques dans son siècle. Une critique de la vie publique en général (à travers les âges et les espaces) occupe ainsi une place plus importante dans le dernier Montaigne (1588-1592). Ces appréciations de la vie publique sont elles-mêmes déterminées et influencées par sa propre expérience des charges publiques.

La question est alors de savoir si l'essai en tant que forme est fondé ou non sur une séparation entre le public et le privé. Le maire et Montaigne - 
et surtout Montaigne après la mairie (1581-1585) -, peuvent-ils vraiment être deux ? Montaigne s'interroge sur les plaisirs de la vie privée à plusieurs reprises dans ses Essais :

[B] J'ayme la vie privée, par ce que c'est par mon chois que je l'ayme, non par disconvenance à la vie publique, qui est, à l'avanture, autant selon ma complexion. J'en sers plus gayement mon prince par ce que c'est par libre eslection de mon jugement et de ma raison, $[\mathrm{C}]$ sans obligation particuliere, $[\mathrm{B}]$ et que je n'y suis pas rejecté ny contrainct pour estre irrecevable à tout autre party et mal voulu. (III, 9, 988)

Servir le prince serait donc un choix plutôt qu'une obligation. Ce passage refuse de mettre en compétition la vie publique et la vie privée. Les deux ne s'opposent pas et l'on ne choisit pas la vie privée par échec de la vie publique. Cette affirmation doit pourtant être prise avec précaution car elle se situe après une période de désillusion politique qui suivit la mairie de Bordeaux. Notons aussi que l'ajout «sans obligation particulière » est plus tardif et marque une rupture définitive avec la vie publique qui, à la Renaissance, est principalement fondée sur le principe d'obligation, voire de servitude, en dépit de ce que peut penser Montaigne. Cette précision a posteriori sur la vie publique est symptomatique d'un changement d'attitude par rapport aux attentes de Montaigne après 1588.

Etrange proposition de suivre son roi par « libre eslection » de son jugement. Une telle idéalisation de la vie publique (tout comme si elle était le prolongement de la vie privée) est pourtant loin de correspondre à la réalité sur le terrain, et Montaigne ne pouvait ignorer ce fait. Son discours s'appuie sur le modèle de l'amitié (La Boétie plus particulièrement) mais il est devenu intenable au fil des ans (et c'est peut-être également pourquoi l'ami s'effacera à son tour des Essais à partir de 1588). Le pouvoir du prince n'est pas un choix mais bien une obligation. Comme nous l'avons vu, ce devoir d'obéissance et d'allégeance est présent dans les Essais de $1580^{17}$.

Si dans un premier temps Montaigne a pu croire que la naïveté affichée sur son visage pouvait le servir dans les offices publics comme dans les ambassades, il reconnaît après 1585 que son style « privé » ne peut convenir aux négociations publiques :

17 Nous avons analysé le rapport de Montaigne à l'allégeance dans "Servitude et allégeance chez La Boétie et Montaigne ", in Figure di 'servitù' e 'dominio' nella cultura filosofica europea tra Cinque e Seicento, dir. Nicola Panichi, Florence, Le Lettere, 2009, p. 25-43. 
[B] J'ay naturellement un stile comique et privé, mais c'est d'une forme mienne, inepte aux negotiations publiques, comme en toutes façons est mon langage : trop serré, desordonné, couppé, particulier; et ne m'entens pas en lettres ceremonieuses, qui n'ont autres substance que d'une belle enfileure de paroles courtoises. Je n'ay ny la faculté, ny le goust de ces longues offres d'affections et de services. Je n'en crois pas tant, et me desplaist d'en dire guiere outre ce que j'en crois. (I, 50, 252)

On comprend alors qu'à la relecture de l'avis au lecteur lors de la publication parisienne des Essais de 1588 Montaigne se soit reconnu - certes d'une façon un peu différente - dans sa défense de l'écriture privée. Non sans ironie, l'avis au lecteur n'est pourtant plus un désir mais bien une réalité qui blesse. La perspective d'une carrière publique est désormais derrière lui et il est certain que la vie privée s'impose faute de mieux. C'est tout ce qui reste à Montaigne, et c'est dans ce sens que les chapitres du troisième livre optent pour l'écriture de l'expérience individuelle, de l'introspection et de la distanciation par rapport au domaine du politique et à la vie publique en général. La division entre le public et le privé commence à faire sens dans la mesure ou elle est devenue réalité, non par choix mais par le jeu de circonstances politiques défavorables à Montaigne.

Dans un long développement rédigé à l'occasion de la publication du troisième livre des Essais, Montaigne fait l'histoire de son rapport compliqué aux offices et à la vie publique. Selon lui, la vie publique n'a jamais représenté un choix, mais un milieu dans lequel il baigna depuis sa tendre enfance:

[B] Tout ce mien proceder est un peu bien dissonant à nos formes; ce ne seroit pas pour produire grands effets, ny pour y durer ; l'innocence mesme ne sçauroit ny negotier entre nous sans dissimulation, ny marchander sans manterie. Aussi ne sont aucunement de mon gibier les occupations publiques ; ce que ma profession en requiert, je l'y fournis, en la forme que je puis la plus privée. Enfant, on m'y plongea jusques aux oreilles, et il succedoit ; si m'en desprins-je de belle heure. J'ay souvant depuis evité de m'en mesler, rarement accepté, jamais requis ; tenant le dos tourné à l'ambition ; mais sinon comme les tireurs d'aviron qui s'avancent ainsin à reculons, tellement toutesfois que, de ne m'y estre poinct embarqué, j'en suis moings obligé à ma resolution qu'à ma bonne fortune ; car il y a des voyes moings ennemyes de mon goust et plus conformes à ma portée, par lesquelles si elle m'eut appelé autrefois au service public et à mon avancement vers le credit du monde, je sçay que j'eusse passé par dessus la raison de mes discours pour la suyvre. (III, 1, 795)

On aurait donc forcé Montaigne dans la vie publique. On pensera ici évidemment à l'influence du père de Montaigne. Comme quand il fallut expliquer la traduction de la Théologie naturelle de Raymond Sebond au début de l' « Apologie de Raymond Sebond », Montaigne blâme un père autoritaire qui a bon dos. Michel fait soi-disant de la politique « à reculons », essayant de 
modeler la vie publique à partir de sa vie privée. Nous avons le sentiment que Montaigne, à partir de 1585 et surtout après 1588, a finalement réussi à séparer les deux dans ses Essais. L'étude des essais en politique de Montaigne prouve néanmoins que « cette separation bien claire » voulue et constatée est plus une invention littéraire qu'une réalité biographique. Créée assez tardivement lors de la dernière carrière de Montaigne - une carrière d'écrivain -, la segmentation artificielle entre vie privée et vie publique permettra à Montaigne de s'affirmer comme moraliste et de juger la politique de l'extérieur : « [B] Le bien public requiert qu'on trahisse et qu'on mente [C] et qu'on massacre; [B] resignons cette commission à gens plus obeissans et plus soupples » (III, 1, 791). Sa résignation à la vie privée ne lui donne malheureusement plus aucun moyen d'influencer des pratiques qu'il réprouve. Juger la vie publique sans se donner les moyens d'y participer est une position assez scabreuse dont le meilleur modèle historique reste Socrate ${ }^{18}$.

Montaigne aurait pu choisir de rédiger des ouvrages où l'histoire de son temps aurait occupé une place plus importante. On a même suggéré qu'il se serait livré à cet exercice très à la mode à son époque ${ }^{19}$. Dans le chapitre « De la force de l'imagination ", Montaigne anticipe ce que cela aurait pu donner : « [C] Aucuns me convient d'escrire les affaires de mon temps, estimans que je le voy d'une veuë moins blessée de passion qu'un autre, et de plus pres, pour l'accez que fortune m'a donné aux chefs de divers partis » $(\mathrm{I}, 21,106)$. Cet aveu en dit long sur ce qu'auraient pu devenir les Essais. De façon peutêtre plus significative, Montaigne admet aussi l'existence d'une vie politique chargée, une vie publique fondée sur un modèle d'allégeance (ambassadeur, négociateur, intermédiaire, homme de l'ombre) où la parole directe occupe une place bien plus importante que l'écrit. C'est là une autre conception de la vie publique. Montaigne n'est pas un Monluc ou un D'Aubigné, sa vie publique a laissé peu de documents pour les historiens. Mais faut-il pour autant croire que la vie privée l'emporta finalement chez Montaigne, lui permettant ainsi de passer dans la postérité ?

Après 1588, les Essais ne sont plus compatibles avec la pratique du politique de son temps, surtout à la lumière des événements qui suivent les assassinats du duc de Guise et de Henri III. La situation bien particulière de la Guyenne doit inévitablement être prise en considération pour expliquer les

18 Sur ce point voir notre étude, "Le Socrate de Montaigne ou 'la science de s'opposer' », in Le Socratisme de Montaigne, Le Socratisme de Montaigne, dir. Thierry Gontier et Suzel Mayer, Paris, Classiques Garnier, 2010), p. 87-103.

19 M. C. Smith, "Lost Writings by Montaigne ", Bibliothèque d'Humanisme et Renaissance, t. XLIX, $\mathrm{n}^{\circ} 2,1987$, p. 309-318. 
déconvenues de Montaigne en politique. L'auteur des Essais aura essayé de se rendre utile et de se faire rappeler au bon souvenir du roi et de Matignon qui verrouille Bordeaux et tente de contrôler, bon gré mal gré, la région. Nous sommes aux antipodes des premiers Essais fondés sur une conviction que la politique et la diplomatie pouvaient être marquées par une âme forte pleine de convictions et qui désirait imposer une pratique nouvelle.

Entre 1588 et 1592 Montaigne offre une nouvelle perspective pour son entreprise d'auteur. L'avertissement au lecteur exprime non plus un souhait mais désormais une réalité. On ne peut pourtant pas exprimer une désillusion sans conserver les traces de pratiques désavouées qui serviront à comprendre le parcours hors du politique. Henri III n'a pas tenu ses promesses de 1580. Marguerite de Valois, connaissant mieux que quiconque ce monarque, parlait en ces termes de son frère : " le Roy estoit de telle humeur qu'il s'offençoit non seulement des effets, mais des imaginations, et qu'estant resolu en ses opinions, sans s'arrester à aucun advis ni d'elle ni d'autre, il executoit tout ce qui luy venoit en fantaisie $»^{20}$. Henri III est une véritable girouette et change fréquemment d'avis. Montaigne choisit de ne pas supprimer ses premières vues du politique, mais de les tempérer. La réalisation de l'échec représente un lent parcours pour notre auteur. Supprimer plus de la moitié de son texte aurait certes rendu la position du « dernier Montaigne » plus cohérente, mais les erreurs d'une vie font partie intégrante de l'écriture du sujet. Comme il l'assume désormais, Montaigne " faict des Essais qui ne sauroit faire des effects » (III, 9, 992). Les effets il les conserve pour l'avertissement au lecteur, ce texte presque inchangé mais qui ne manque pas de produire des effets différents sur la lecture de ses Essais.

À la fin de l'année 1590 l'autorité du roi en Guyenne n'est guère respectée. La Ligue s'empare de plusieurs places fortes. Le Parlement de Bordeaux adresse une lettre au roi sur l'état lamentable de la province et se plaint de la « ruyne et subversion » qui règne en Guyenne ${ }^{21}$. Les arrêts royaux n'ont plus grand effet. La confusion règne et les Parlementaires n'hésitent pas à décrire la situation politique comme anarchique. La Ligue possède une influence considérable dans le milieu. Le Parlement de Bordeaux délibérera longtemps pour savoir s'il devait reconnaître le roi de Navarre comme roi de France.

20 Mémoires de Marguerite de Valois, reine de France et de Navarre, in Collection complète des mémoires relatifs à l'histoire de France, éd. Claude Bernard Petitot, Paris, Foucault, 1823, t. XXXVII, p. 152-153.

21 Archives Historiques de Guyenne, 30 octobre 1590, t. VI, n LXXIV, p. 210. Une nouvelle lettre est envoyée au roi le 15 novembre exprimant les mêmes craintes. Après la chute de Rions au ligueurs puis d'Ayre de Mezin et de Villandrault, ils considèrent que Bordeaux est, non sans exagération, « desjà comme assiégée » et que la guerre est déjà à ses portes. 
Une motion présentée durant « l'interrègne » proposait de déclarer Henri de Bourbon, roi de Navarre, " incapable d'estre roy de France »22. La motion fut mise en délibéré le 22 septembre et interrompue le 26 car la cour jugeait qu'elle avait finalement « peu de moyen qu'il y avoit de faire recognoistre Henry de Bourbon, roy de Navarre, pour roy de France en la Guyenne $»^{23}$. À la fin octobre le parlement n'avait toujours pas décidé s'ils doivent utiliser le mot « roy » ou simplement le mot « magesté » pour parler du roi de Navarre. Un vote s'ensuivit « et passa à vingt et cinq contre vinct qu'on useroit dans les mémoires du mot de magesté pour ne passer coup à la délibération interrompue et cependant l'honorer du titre ${ }^{24}$.

Comment relire ses propres Essais dans un tel contexte politique ? Comment imaginer un nouveau lecteur? On peut dire qu'après 1588 Montaigne a sa carrière politique derrière lui, ou du moins qu'il n'a plus les prétentions du début des années 1580. Si les diverses éditions des Essais (1580, 1582, 1588, et Exemplaire de Bordeaux) sont marquées par des étapes politiques très différentes dans leur nature et leurs intentions, elles n'ont pourtant rien à voir avec une quelconque évolution de la pensée de Montaigne et une victoire de la vie privée sur la vie publique. Peut-on d'ailleurs parler d'une politique éditoriale (au singulier) dans le cas de la publication des œuvres de Montaigne du vivant de l'auteur ? Par politique éditoriale nous entendons à la fois l'intention calculée qui consiste à élaborer à un moment précis une stratégie de publication répondant à des fins précises, mais aussi et surtout le projet mis sur pied par Montaigne afin d'utiliser sa propre carrière d'écrivain à des fins politiques. C'est donc dans cette double acception du mot «politique » que nous proposons de relire l'avertissement au lecteur avec toutes ses ambiguïtés et contradictions par rapport aux aspirations et pratiques de l'auteur.

Comme on le sait, Montaigne devint auteur par accident, c'est du moins ce qu'il affirme. Méfions-nous pourtant des légendes. Al'en croire, Montaigne se soucie peu de son public et n'écrit que pour exister après sa mort dans la mémoire de ses parents et amis proches. Il n'est d'ailleurs pas vraiment un auteur : « Les autheurs se communiquent au peuple par quelque marque particuliere et estrangere ; moy, le premier, par mon estre universel, comme Michel de Montaigne, non comme grammairien, ou poëte, ou jurisconsulte » (III, 2, 805). Son livre est tout autre et l'écriture impréméditée représente un des topoi des Essais. Il faudrait donc vérifier si les déclarations répétées 
de Montaigne sur ces publications privées et particulières (pour son père en ce qui concerne la Théologie naturelle, pour son ami disparu dans le cas de la Mesnagerie de Xenophon, ou tout simplement « voué à la commodité particulière de [s]es parens et amis » pour ce qui est des Essais), peuvent être validées au niveau de la pratique. L'interprétation des Essais passe inévitablement par une meilleure compréhension de l'horizon d'attente de son auteur, mais aussi par la réception anticipée d'un lecteur réel ou imaginé.

Si une philosophie se dégage indubitablement des Essais, elle a peut-être plus à voir avec la manipulation constante effectuée par Montaigne entre le particulier (vie privée) et l'universel (vie publique) que sur le contenu de tel précepte moral ou dogme des écoles antiques. La temporalité de l'écriture et celle des événements ne sont pas en synchronie dans les Essais et Montaigne joue constamment sur cette image d'un livre privé (hors de toute temporalité historique) alors que les Essais sont conçus - comme nous avons essayé de le démontrer - comme un objet lui permettant précisément l'accès à la vie publique. Difficile en effet d'imaginer les Essais comme un objet entièrement privé (comme l'affirme Montaigne dans son avis "Au lecteur »). Cette philosophie du quotidien et du temps présent qui ressort des Essais ne saurait mettre à l'écart les considérations publiques et historiques qui, comme nous l'avons vu, rythment toujours l'écriture d'une vie bien particulière. Le privé ne peut être compris hors du public : c'est peut-être là la meilleure leçon de l'histoire et, de façon plus générale, de toute démarche philosophique. 